\title{
Presencia de Linfocitos T Reguladores en Periodontitis Crónica
}

\author{
Presence of T Regulatory Cells in Chronic Periodontitis
}

\author{
Carré L ${ }^{1}$, Franco ME², Henríquez L³ , García-Sesnich J4 , Dutzan N ${ }^{5}$, Aguillón JC ${ }^{6}$, Gamonal J7
}

\begin{abstract}
RESUMEN
La enfermedad periodontal requiere de un hospedero susceptible para su desarrollo y progresión. Dentro de las características del hospedero se encuentra la respuesta $T$ reguladora, que otorga tolerancia frente a antígenos propios, participa durante las enfermedades infecciosas limitando el daño tisular, sin disminuir la respuesta antibacteriana. El presente estudio tiene por objetivo determinar la presencia, reclutamiento y función de Tregs en pacientes con periodontitis crónica. En 10 biopsias de tejido periodontal sano y con periodontits crónica se realizó inmunohistoquímica para marcadores (CD4, CD25, Foxp3), quimioquinas (CCL17, CCL22) y citoquinas (TGF- $\beta$, IL-10) de Tregs. Además de Western-Blot para detectar las citoquinas. Los resultados obtenidos sugieren una posible asociación entre células Tregs y la infección periodontal, ya que se confirma su reclutamiento y presencia. Sin embargo, son necesarios más estudios del posible desbalance con su contraparte pro-inflamatoria Th17, que expliquen en parte la compleja etiopatogenia de la enfermedad periodontal.
\end{abstract}

Rev. Clin. Periodoncia Implantol. Rehabil. Oral Vol. 3(3); 128-131, 2010.

Palabras clave: Linfocitos T reguladores, periodontitis, reclutamiento.

\begin{abstract}
Periodontal disease requires a susceptible host to initiation, development and progression. T regulatory response is one of these inmunoregulatory characteristics of the susceptible host, which provide tolerance, tissular protection during infection without impairing the control of periodontopathogens. The aim of this study is to determinate the presence, homing and function of T regulatory cells (Tregs) in patients with chronic periodontitis. Ten biopsies were taken from pockets, the presence of Tregs markers (CD4, CD25, Foxp3), chemokines (CCL17, CCL22) and cytokines (TGF- $\beta$, IL-10) were determinate by immunohistochemistry. Cytokines also were detected with Western-Blot. Our results suggest a possible association between Tregs and periodontal infection, confirming homing and presence of Tregs. However, further studies are required to determine the possible imbalance with pro-inflammatory part Th17, that might explain the complex etiopathogenesis of periodontal disease.
\end{abstract}

Rev. Clin. Periodoncia Implantol. Rehabil. Oral Vol. 3(3); 128-131, 2010.

Key words: T regulatory cells, periodontitis, homing.

\section{INTRODUCCIÓN}

La periodontitis es una enfermedad de etiología infecciosa y de naturaleza inflamatoria que afecta los tejidos de inserción del diente, la cual para desarrollarse necesita de patógenos periodontales específicos además de un hospedero susceptible ${ }^{(1,2)}$.

Los periodontopatógenos son los agentes etiológicos de la enfermedad periodontal, sin embargo, un importante determinante de la progresión y desarrollo de la enfermedad es la respuesta inmune del hospedero ${ }^{(3)}$, puesto que la naturaleza de la respuesta inflamatoria influye en el carácter destructivo de la enfermedad(4).

Se han propuesto muchos modelos para intentar explicar la patogénesis de la enfermedad y que, a la vez, concuerden con la presencia de linfocitos ${ }^{(4)}$, entre ellas el balance entre las respuestas autoinmunes y los mecanismos regulatorios ${ }^{(3)}$. Los linfocitos $T$ reguladores (Tregs) migran y se acumulan a nivel de tejidos inflamados, tal como los tejidos gingivales, que en periodontitis presentan un infiltrado inflamatorio(5).
Existe una pequeña cantidad de estudios que reportan la presencia de Tregs y su relación con enfermedades periodontales ${ }^{(3-6)}$. Éstos han analizado en un número reducido de pacientes con periodontitis crónica, muestras de sangre periférica y biopsias de tejido gingival analizándolas mediante citometría de flujo, reacción de la cadena de polimerasa en tiempo real (RT-PCR) e inmunohistoquímica.

Los Tregs se caracterizan por la presencia de marcador de superficie CD4, pues son linfocitos T ayudadores. En su membrana cuentan con una alta expresión de CD25, el receptor de IL-2, además de la expresión constitutiva del factor de transcripción Foxp3, que comanda el patrón de expresión y funcionalidad de estos linfocitos. Estos tres elementos son necesarios para determinar la presencia de Tregs ${ }^{(7)}$.

Para mediar una respuesta efectiva los leucocitos deben encontrar señales que los guien a los sitios de infección y/o inflamación ${ }^{(8)}$ Las proteínas encargadas de este reclutamiento en el caso de los Tregs son las quimioquinas CCL17 y CCL22 $2^{(6-8)}$. Se piensa que éstas juegan un rol importante en la inmunopatogénesis de las enfermedades

1. Cirujano Dentista. Alumna Magíster en Cs. Odontológicas Mención Periodontología. Laboratorio de Biología Periodontal. Facultad de Odontología, Universidad de Chile. Chile.

2. Tecnólogo Médico. Profesora Asistente, Departamento de Patología Oral. Facultad de Odontología, Universidad de Chile. Chile.

3. Técnico Paramédico. Laboratorio de Biología Periodontal. Facultad de Odontología, Universidad de Chile. Chile.

4. Bioquímico. Laboratorio de Biología Periodontal. Facultad de Odontología, Universidad de Chile. Chile.

5. Cirujano Dentista. Magíster en Cs. Odontológicas Mención Periodontología. ProfesorAsistente Área de Periodoncia, Laboratorio de Biología Periodontal, Departamento de Odontología Conservadora. Facultad de Odontología, Universidad de Chile. Chile.

6. Bioquímico. Doctor en Bioquímica. Director del Programa Disciplinario de Inmunología. Facultad de Medicina, Universidad de Chile. Chile.

7. Cirujano Dentista. Especialista en Periodoncia. Magíster y Doctor en Cs. Odontológicas. Director del Departamento de Odontología Conservadora. Facultad de Odontología, Universidad de Chile. Chile.

Correspondencia autor: Lisette Carré Benzi. lizzycarre@gmail.com. Laboratorio de Biología Periodontal. Facultad de Odontología, Universidad de Chile. Sergio Livingstone Pohlhammer 943. Independencia. Santiago, Chile.

Financiamiento: Proyecto FONDECYT 1090046. Trabajo recibido el 07/12/2010. Aprobado para su publicación el 17/12/2010. 
periodontales, pudiendo atenuar la severidad de la enfermedad(8).

Las citoquinas que permiten la disminución de la respuesta inmune, producidas por los Tregs, son IL-10 y TGF- $\beta$. IL-10 es considerada como una citoquina antiinflamatoria por excelencia, y ha sido confirmada su importancia al atenuar la severidad de la enfermedad mediante mecanismos como diminuir la susceptibilidad a $p$. gingivalis, acción directa sobre INF-y e IL-17, metaloproteínasas y el sistema de receptor activador para el factor nuclear $\mathrm{K} B(\mathrm{RANK})^{(9)}$. En tanto, TGF- $\beta$ es un factor de crecimiento asociado principalmente a fibroblastos a nivel del tejido conjuntivo, además de ser necesario para la diferenciación de los linfocitos y la acción inmunoregulatoria de los Tregs ${ }^{(7)}$.

El presente estudio se enfoca en mostrar la presencia de Tregs y sus citoquinas en biopsias de tejido periodontal sano y con periodontitis crónica, con el objetivo de confirmar su distribución tanto en salud como enfermedad.

\section{MATERIAL Y MÉTODOS}

Se reclutaron individuos que acuden a la Facultad de Odontología de la Universidad de Chile y al Área de Periodoncia del Complejo Hospitalario Norte perteneciente al Servicio de Salud Metropolitano Norte CDT Dra. Eloísa Díaz. Se seleccionó a 10 pacientes con periodontitis crónica y 10 individuos sanos.

Los criterios de inclusión para los pacientes con periodontitis crónica fueron: edad mayor o igual a 35 años con enfermedad periodontal crónica de magnitud moderada a avanzada ${ }^{(10)}$. Se consideró como periodontitis moderada a avanzada aquellos sujetos con al menos 4 sitios de profundidad al sondaje mayor a $5 \mathrm{~mm}, 6$ sitios con pérdida de inserción mayor a $3 \mathrm{~mm}$ y evidencia radiográfica de destrucción ósea alveolar. En el grupo control se incluyeron individuos sistémica y gingivalmente sanos, voluntarios, sometidos a cirugía de alargamiento coronario o exodoncia por indicación ortodóntica o exodoncia de terceros molares. Se excluyeron los pacientes que hayan recibido algún tipo de terapia periodontal, medicación antibiótica 3 a 6 meses previos al estudio, que fumen, que tengan antecedentes de enfermedad autoinmune, infecciosa y/o inflamatoria en el transcurso de los últimos 6 meses, o la presencia de estados fisiológicos como el embarazo y afecciones sistémicas que pudieran afectar el desarrollo de la enfermedad periodontal, como diabetes.

Toda persona incluída en este estudio firmó un consentimiento informado, el cual fue aprobado por el Comité de Ética de la Facultad de Odontología de la Universidad de Chile y por el Fondo Nacional de Desarrollo Científico y Tecnológico (FONDECYT).

Se realizó una biopsia a aquellos sitios con profundidad al sondaje mayor o igual a $5 \mathrm{~mm}$ en los pacientes o el sitio a intervenir en los sujetos sanos. Previa anestesia local del sitio seleccionado, retiro de placa supra gingival y aislamiento relativo con tórulas de algodón, se realizó la toma de biopsia. Con un bisturí hoja $\mathrm{N}^{\circ} 10$ ó 15 se procede a realizar una incisión a 1-2 mm desde el margen gingival, dirigida hacia la cresta alveolar, siguiendo el eje mayor del diente, de modo que la biopsia contenga epitelio y tejido conectivo.

Una vez tomada la biopsia se lavó con $\mathrm{NaCl} 0.15 \mathrm{M}$ estéril para eliminar detritus y coágulos sanguíneos. Se mantuvieron en formalina buffer $4 \%$ para realizar inmunohistoquímica o en medio de transporte RPMI $1640\left(4^{\circ} \mathrm{C}\right)$ para su posterior almacenamiento seco a $-80^{\circ} \mathrm{C}$.

Las biopsias de tejido gingival fijadas en formalina buffer $4 \%$ se deshidratan e incluyen en parafina, así se almacenaron. Se utilizaron anticuerpos mono clonales anti-humanos contra, CD4 (CD4 Mouse Monoclonal Antibody Cat $N^{\circ}$ NCL-CD4-1F6, Novocastra $\left.®\right)$ y CD25 (Antibody to Interleukin-2 Receptor Cat. No VP-C340, Vector Laboratories), Foxp3 (Foxp3 Antibody Cat $N^{\circ}$ ab20034, Abcam) como marcadores de Tregs. Para detectar las quimioquinas se utilizaron anticuerpos CCL17 (Anti-human CCL17/TARC Antibody, Cat № AF364 R\&D Systems) y CCL22 (Rabbit Polyclonal Anti-ABCD-1/CCL22, Cat No 1861-1, Epitomics).

Luego, se cortan secciones de $4 \mu \mathrm{m}$, las cuales se desparafinan y rehidratan mediante protocolo standard. El procesamiento de los tejidos comienza con el bloqueo de la peroxidasa y desemascaramiento antigénico con Buffer Citrato $0.01 \mathrm{M} \mathrm{pH} 6$. Luego se procedió a marcar con el anticuerpo seleccionado, el cual se incubó durante la noche a $4^{\circ} \mathrm{C}$. Para la tinción se utilizó un método estándar de avidina-biotina peroxidasa (Vectastain, Elite ABCKit, Vector; Vector Laboratories Inc.,
Burlingame, CA, USA) según las indicaciones del fabricante. Se revela con el kit DAB para peroxidasa (Immpact DAB Kit for Peroxidase; Vector Laboratories) como cromógeno y contratinción nuclear hematoxilina. En cada procedimiento de tinción se realizó un control negativo sin los anticuerpos primarios. Biopsias de amígdalas humanas se utilizaron como controles positivos.

También, se detectó la presencia de las citoquinas IL-10 (Monoclonal Anti-human IL-10 Antibody, Cat N ${ }^{\circ}$ MAB2171, R\&D Systems Inc.) y TFG- $\beta$ (Mouse Monoclonal TB21 to TGF Beta 1, Cat Nº ab27969, Abcam), en biopsias de tejido periodontal de individuos sanos y pacientes con periodontitis mediante inmunohistoquímica, como se describe previamente, y mediante la técnica de Western-Blot.

De las biopsias guardadas en seco a $-80^{\circ} \mathrm{C}$ se prepararon unos homogenizados mediante un homogenizador mecánico y su dilución a 2 $\mathrm{ml}$ con cloruro de sodio al $0.9 \%$. La medición de proteínas totales se realizó mediante el método del ácido bisciconitico (Micro BCA Protein Assay Reagent Kit, Pierce Biotechnology, Rockford, USA), siguiendo las indicaciones del proveedor.

Para realizar el Western-Blot, se prepararon geles de trisglicina al $15 \%$ cargando muestras de individuos sanos y enfermos, más control de peso molecular (Benchmark Presetained Protein Ladder, Cat N¹07478-010, Invitrogen). Se corre el gel, posteriormente se transfiere a una membrana de PDVF la cual bloqueada con suero bovino fetal al 3\% (BSA-50, Rockland Inc.) y se incuba con el anticuerpo primario contra la citoquina a detectar, durante toda la noche a $37^{\circ} \mathrm{C}$ con agitación. Al día siguiente se le adiciona el anticuerpo secundario (Stabilized Goat Anti Mouse IG Peroxidase Conjugated Cat $\mathrm{N}^{\circ} 32430$, Thermo Scientific), y se revela mediante quimioluminiscencia (SuperSignal West Femto, Pierce Biotechnology, Rockford, USA) en placas radiográficas.

\section{RESULTADOS}

Las características clínicas de los sujetos incluídos en el estudio se detallan en la Tabla 1. Brevemente, podemos observar en la muestra que los sitios periodontales de pacientes con periodontitis tienen mayor pérdida de destrucción que los sitios provenientes de sujetos sin enfermedad.

Tabla 1. Datos clínicos de los pacientes del estudio. Porcentaje de mujeres del estudio, promedio de edad, desviación estándar y profundidad al sondaje promedio del sitio biopseado.

\begin{tabular}{|l|c|c|}
\hline & Sanos (N=10) & Periodontitis (N=10) \\
\hline Mujeres (\%) & $40 \%$ & $50 \%$ \\
\hline Edad \pm DS & $35.75 \pm 13.23$ & $45.66 \pm 5.81$ \\
\hline Profundidad Sondaje Sitio & $1.85 \pm 0.69$ & $6.16 \pm 1.61$ \\
\hline
\end{tabular}

La presencia de los Tregs con sus marcadores de superficie e intracelular fue evidente a nivel del infiltrado inflamatorio de los pacientes con periodontitis. Se encontraron grandes cantidades de células CD4+ en todos los infiltrados inflamatorios. Además, se observó una fuerte activación de linfocitos representado por el marcaje CD25+, su alta expresión se asocia con los Tregs. Los Tregs podrían identificarse como células que presentan morfología compatible con linfocitos con marcaje más intenso para CD25, que podemos distinguir como focos al interior del infiltrado (Figura 1). 


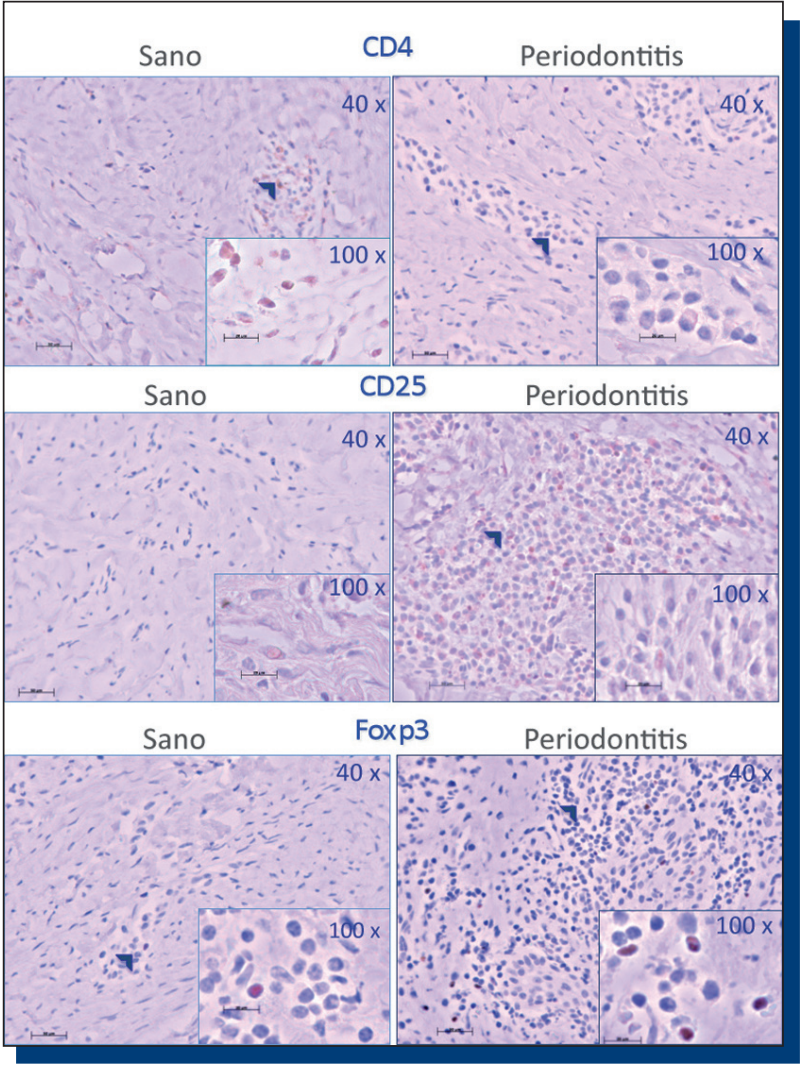

Figura 1. Inmunohistoquímica de biopsias de tejido periodontal marcadas con anticuerpos para detectar la presencia de Tregs (CD4+CD25+Foxp3+) con aumentos de 40X y $100 \mathrm{X}$.

Por otra parte, en los individuos sanos la presencia de los marcadores Foxp3 y CD4 fue menor, siendo muy difícil de detectar CD25. La morfología de las células asociadas a estos tres marcadores en sujetos sanos fue bastante variada, asociada a células inmunes como linfocitos que presentan un núcleo más pequeño, al compararlos con los de enfermos, y por ende menos activados (Figura 1).

Las quimioquinas CCL17 y CCL22, que reclutan a nivel de tejido selectivamente Tregs, se encontraron en ambas condiciones clínicas (Figura 2). En tejidos sanos su distribución fue diversa, tanto a nivel de tejido conjuntivo como infiltrados aislados. En cambio, en periodontitis crónica se observó una marcada presencia en los sitios de inflamación.

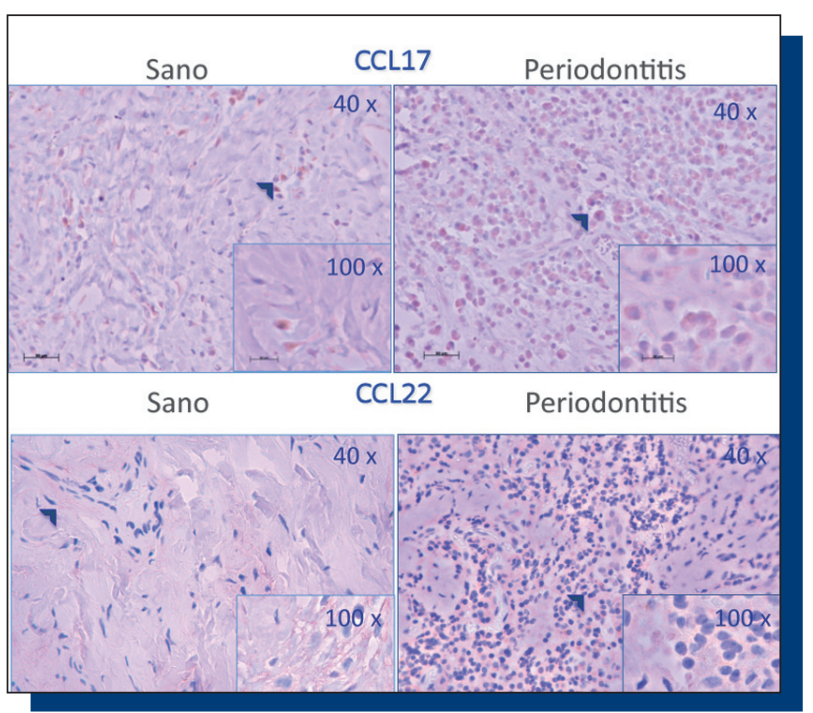

Figura 2. Inmunohistoquímica de biopsias de tejido periodontal marcadas con anticuerpos contra quimioquinas que reclutan Tregs, CCL17 y CCL22, con aumentos de 40X y 100X.
Las citoquinas IL-10 y TGF- $\beta$ fueron detectadas exitosamente mediante inmunohistoquímica y Western-Blot (Figura 3), con una presencia muy similar al comparar los tejidos sanos y enfermos. En los cortes histológicos analizados estas citoquinas se distribuyen ampliamente, en sanos y enfermos se asocian a las células infiltrantes. Sin embargo, en los enfermos se observa menor marca en el tejido conjuntivo que en sujetos sanos.

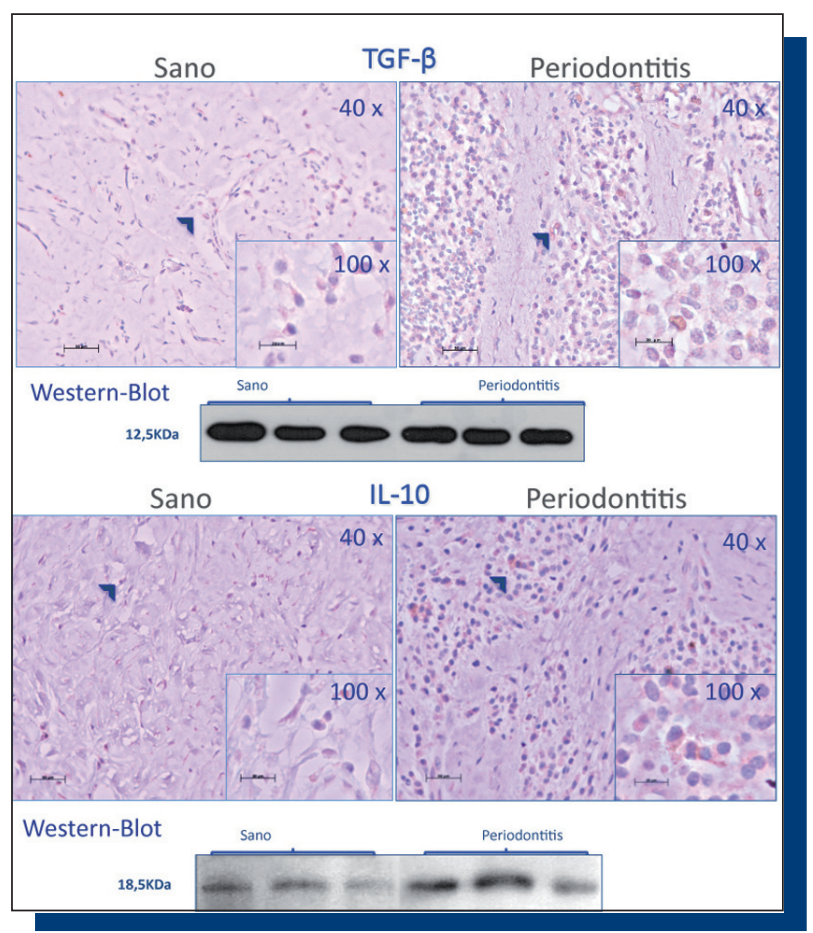

Figura 3. Inmunohistoquímica y Western-Blot de biopsias de tejido periodontal de citoquinas asociadas a la función de Tregs, TGF- $\beta$ e IL-10.

Mediante Western-Blot, la intensidad y tamaño de las bandas es muy similar entre los sujetos sanos y los enfermos (Figura 3). Sin representar una cuantificación, a nivel visual, podemos inferir una tendencia a la igualdad en cuanto a la cantidad de IL-10 y TGF- $\beta$ en las biopsias analizadas.

\section{DISCUSIÓN}

La importancia de los Tregs ha sido estudiada a nivel de enfermedades autoinmunes, infecciosas e inflamatorias y condiciones fisiológicas como el embarazo, por su rol tanto en tolerancia a lo propia como en la evasión a la respuesta inmune ${ }^{(11)}$.

En periodontitis, los grandes infiltrados inflamatorios presentes se encuentran con gran presencia de linfocitos $T$ ayudadores (CD4+), linfocitos activados (CD25+) y con programa genético de Tregs (Foxp3+); todo esto en conjunto nos muestra una abundante presencia de Tregs en las biopsias examinadas. Se ha encontrado aumento en el número de Tregs en sujetos con periodontitis crónica en comparación con sanos ${ }^{(6,12)}$ o con gingivitis ${ }^{(3)}$, a nivel de tejido, en un posible intento de restringir la respuesta del hospedero( ${ }^{(9)}$, lo cual concuerda con nuestro estudio.

En un reciente estudio en murinos ${ }^{(13)}$, en periodontitis experimental, se observó una menor progresión de la enfermedad periodontal cuando los Tregs se encuentran funcionales. El comprobar la presencia de Tregs en individuos sanos podría estar en relación con la importancia de éstos en la homeostasis de los tejidos frente a un desafío bacteriano dentro de límites de salud( ${ }^{(9)}$. Además, la amplia distribución de sus citoquinas en encías sanas, tanto en tejido como en los pocos infiltrados que podemos encontrar, nos indicarían que existen procesos inmunes activos que requieren del control de los Tregs para mantener la salud periodontal.

Una posible explicación del control de la cinética de los Tregs es el patrón de quimioquinas expresados en los tejidos ${ }^{(8,9)}$. Los Tregs migran usualmente atraídos por las quimioquinas CCL1, CCL17 
y CCL22, ya que expresan los receptores CCR4 y CCR8 ${ }^{(6-8)}$. Cardoso et al. $(2008)^{(6)}$ confirmaron que en biopsias de saco periodontal hay un aumento significativo de estas 5 moléculas comparado con un control sano, y que por lo tanto, existe un homing de Tregs hacia estos sitios. Nosotros, mediante inmunohistoquímica, confirmamos que CCL17 está principalmente asociado con epitelio, células endoteliales, fibroblastos y linfocitos, mientras que CCL22 aparentemente es producido por células con morfología compatible con grandes leucocitos como los histocitos. Así, inferimos que la localización apropiada es indispensable para la función de los Tregs in vivo y el comportamiento migratorio de sus subtipos influencia su capacidad supresora ${ }^{(8)}$.

Su acción protectora de los tejidos en presencia de inflamación, limitando la destrucción tisular mediante citoquinas como TGF- $\beta$ e IL10 o acción celular directa, los hacen críticos en la progresión de las enfermedades de naturaleza inmune-inflamatorias ${ }^{(9)}$. TGF- $\beta$ tiene acción sobre los fibroblastos presentes en el tejido conectivo periodontal, aumentando la formación de colágeno, con correlación positiva entre la cantidad de fibras colágenas y su presencia, así no solamente afecta a la respuesta $T$ efectora sino que limita el daño estructural de este promoviendo la cicatrización ${ }^{(9,14)}$.

Según nuestros resultados, existe una presencia similar de IL-10 y TGF- $\beta$, sin embargo, se hace necesario cuantificar los niveles de ambas proteínas. En otros estudios se han encontrado niveles aumentados de ambas a nivel de RNA al comparar periodontitis con individuos sanos ${ }^{(6)}$.
Se ha propuesto que los Tregs se asocian con menor progresión de la enfermedad ${ }^{(6)}$, incluso se han propuesto vacunas contra la periodontits con Tregs estimuladas con antígenos específicos capaces de producir IL-10 y TGF- $\beta$, de manera de inducir tolerancia o función efectora $^{(15)}$. El balance entre disminuir el daño colateral de la respuesta inmune frente a la infección y la perpetuación de esta en el tiempo ${ }^{(1)}$ es controversial y podría tener influencia en la presentación clínica e histológica de la periodontitis ${ }^{(16-18)}$.

Es razonable asumir que la susceptibilidad a la destrucción de tejido está determinado, al menos en parte, por el balance entre la respuesta inmune mediada por linfocitos autorreactivos y los mecanismos regulatorios mediados por Tregs ${ }^{(16,19)}$, sin existir aún un estudio con este enfoque en periodontitis que nos muestre si existe un desbalance que podría explicar el quiebre de la homeostasis.

La complejidad de la respuesta inmune-inflamatoria en las enfermedades periodontales ha sido ampliamente estudiada, proponiendose múltiples hipótesis al respecto. Un eje novedoso es el del balance entre la autoinmunidad y tolerancia, respresentada por las respuestas Th17 y T reguladora, respectivamente.

Se necesitan más estudios en relación a los Tregs y cómo su función puede ir guiando las respuestas inmunes en enfermedad periodontal, según el tipo de microorganismos presentes y presentación clínica, para así llegar a alguna aplicación práctica que pueda ser útil para el diagnóstico y/o tratamiento de diferentes patologías.

\section{REFERENCIAS BIBLIOGRÁFICAS}

1. Socransky SS, Haffajee AD. The bacterial etiology of destructive periodontal disease: current concepts. J Periodontol, 1992; 63(4): 322-331.

2. Socransky SS, Haffajee AD, Cugini MA, Smith C, Kent Jr. RL. Microbial complexes in subgingival plaque. J Clin Periodontol, 1998; 25: 134-144.

3. Nakajima T, Ueki-Maruyama K, Oda T et al. Regulatory T-cells infiltrate periodontal disease tissues. J Dent Res, 2005; 84(7): 639-643.

4. Ito H, Honda T, Oda T et al. Gene expression analysis of the CD4+ $T$ cell clones derived from gingival tissues of periodontitis patients. Oral Microbiol Immunol, 2005; 20: 382-386.

5. Okui $\mathrm{T}$, Ito $\mathrm{H}$, Honda T y cols. Characterization of CD4+ FOXP3+ T-cell clones established from chronic inflammatory lesions. Oral Microbiol Immunol, 2008; 23(1): 49-54.

6. Cardoso CR, Garlet GP, Moreira AP et al. Characterization of CD4+ CD25+ natural regulatory $T$ cells in the inflammatory infiltrate of human chronic periodontitis. J Leukoc Biol, 2008; 84(1): 311-318.

7. Vernal R, García-Sanz JA. Th17 and Treg cells, two new lymphocyte subpopulations with a key role in the immune response against infection. Infect Disord Drug Targets, 2008; 8(4): 207-220.

8. Silva T, Garlet G, Fukada $Y$ et al. Chemokines in oral inflammatory diseases: apical periodontitis and periodontal disease. J Dent Res, 2007; 86(4): 306-319.

9. Garlet G. Destructive and protective roles of cytokines in periodontitis: a reappraisal from host defense and tissue destruction viewpoints. J Dent Res, 2010; Aug 25: in press.

10. Armitage G. Periodontal diagnose and classification of periodontal diseases. Periodontology 2000, 2004; 34: 9-21.

11. Mjösberg J, Berg G, Ernerudh J, Ekerfelt C. CD4+ CD25+ regulatory $T$ cells in human pregnancy: development of a Treg-MLC-ELISPOT suppression assay and indications of paternal specific Tregs. Immunology, 2007; 120: 456-466.
12. Dutzan N, Gamonal J, Silva N y cols. Over -expression of fork- head box P3 and its association with receptor activator of nuclear factor-kappa B ligand, interleukin (IL)-17, IL-10 and transforming growth factor-beta during the progression of chronic periodontitis. J Clin Periodontol, 2009; 36: 396-403.

13. Garlet G, Cardoso C, Mariano F y cols. Regulatory T cells attenuate experimental periodontitis progression in mice. J Clin Periodontol, 2010; 37: 591-600.

14. Berglundh T, Liljenberg B, Lindhe J. Some cytokine profiles of T-helper cells in lesions of advanced periodontitis. J Clin Periodontol, 2002; 29: 705-709.

15. Choi J, Seymour G. Vaccines against periodontitis: a Howard-looking review. J Periodontal Implant Sci, 2010; 40: 153-163.

16. Kornman K, Page R, Tonetti M. The host response to the microbial challenge in periodontitis: assembling the players. Periodontol 2000, 1997; 14: 33-57.

17. Seymour G. Importance of the host response in the periodontium. $J$ Clin Periodontol, 1991; 18: 421-426.

18. Zappa U, Reinking-Zappa M, Graf H, Espeland M. Cell populations and episodic periodontal attachment loss in humans. J Clin Periodontol, 1991; 18: 508-515.

19. Sigusch B, Klinger G, Glockmann E y cols. Early-onset and adult periodontitis associated with abnormal cytokine production by activated $T$ lymphocytes. J Periodontol, 1998; 69: 1098-1104. 